\title{
ON EXTENDING SEMIGROUP CHARACTERS
}

\section{by RONALD FULP}

(Received 2nd August 1965; received in revised form 23rd November 1966)

The principal result of this paper is a characterisation of those commutative semigroups $S$ which have the property that each character of each subsemigroup of $S$ can be extended to a character of $S$. This work was partially inspired by the discovery that Theorem 5.65 of (1) is incorrect; it is related to that of Hill, who has obtained a different solution of the problem (2). Warne and Williams proved in (4) that any bounded character defined on an inverse subsemigroup of an inverse semigroup can be extended to the semigroup.

First we prove some preparatory lemmas which give conditions under which a character of a subsemigroup $S$ of a commutative semigroup $T$ can be extended beyond $S$. Note that in considering such a problem there is no loss of generality in assuming that $T$ contains an identity which is contained in $S$ (see, for example, (1)). Wherever appropriate such assumption will be made without specific mention.

Let $T$ denote a commutative semigroup. Suppose that $S$ is a subsemigroup of $T$ and that $\chi$ is a character defined on $S$. Assume that $x$ is an element of $T$ and that $x$ is not in $S$. For any such $x,\{S, x\}$ will denote the subsemigroup of $T$ consisting of all elements of $T$ of the form $s x^{n}$ where $s \in S$ and $n$ is a nonnegative integer. The next two lemmas give sufficient conditions in order that $\chi$ have an extension to the subsemigroup $\left\{S, x^{m}\right\}$ for some positive integer $m$ such that $x^{m} \notin S$.

Lemma 1. If there exists $s_{0} \in S$ such that $s_{0} x \in S$ and $\chi\left(s_{0}\right) \neq 0$, then $\chi$ can be extended to $\{S, x\}$.

Proof. Let $\chi^{+}$denote the function defined on $\{S, x\}$ by

$$
s x^{n} \rightarrow \chi(s) \chi\left(x s_{0}\right)^{n} \chi\left(s_{0}\right)^{-n}
$$

for $s \in S$ and $n$ a non-negative integer. That $\chi^{+}$is well-defined follows from the fact that if $s_{1}$ and $s_{2}$ are in $S$ and $n$ and $m$ are non-negative integers such that $s_{1} x^{n}=s_{2} x^{m}$, then $s_{1}\left(x s_{0}\right)^{n} s_{0}^{m}=s_{2}\left(x s_{0}\right)^{m} s_{0}^{n}$ and

$$
\chi\left(s_{1}\right) \chi\left(x s_{0}\right)^{n} \chi\left(s_{0}\right)^{m}=\chi\left(s_{2}\right) \chi\left(x s_{0}\right)^{m} \chi\left(s_{0}\right)^{n} .
$$

The function $\chi^{+}$is a character of $\{S, x\}$ which extends $\chi$.

Lemma 2. If there exists a positive integer $n$ such that $x^{n} \in S$, then $\chi$ can be extended to $\left\{S, x^{m}\right\}$ for some $m$ such that $x^{m} \notin S$. 
Proof. Suppose there is a character $\chi$ of $S$ which cannot be extended to $\left\{S, x^{m}\right\}$ for any $m$ such that $x^{m} \notin S$. Let $p$ denote the least positive integer such that $x^{p} \in S$. Then for each $y$ in $\{S, x\}$ there exist $s \in S$ and a non-negative integer $n$ less than $p$ such that $y=s x^{n}$.

Case 1. Suppose that $\chi\left(x^{p}\right) \neq 0$. Let $c$ denote a $p$ th root of $\chi\left(x^{p}\right)$. Let $\chi^{+}$ denote the function with domain $\{S, x\}$ defined by $s x^{n} \rightarrow \chi(s) c^{n}$ for $s \in S$ and $n$ an integer such that $0 \leqq n<p$. We show that $\chi^{+}$is well-defined. Suppose $s_{1} x^{n}=s_{2} x^{m}$ for $s_{1}$ and $s_{2}$ in $S$ and for integers $n$ and $m$ such that $0 \leqq n<p$ and $0 \leqq m<p$. For definiteness, assume $m \leqq n$. Now suppose $m<n$. Since $\chi$ cannot be extended to $\left\{S, x^{m+p-n}\right\}$, Lemma 1 and the equation $s_{1} x^{p}=s_{2} x^{m+p-n}$ imply that $\chi\left(s_{2}\right)=0$. Similarly, the equation $\left(s_{1} x^{p}\right) x^{n-m}=s_{2} x^{p}$ implies that $\chi\left(s_{1} x^{p}\right)=0$ and that $\chi\left(s_{1}\right)=0$. It follows that $\chi\left(s_{1}\right) c^{n}=0=\chi\left(s_{2}\right) c^{m}$. If $n=m$, then $s_{1} x^{p}=s_{2} x^{p}$ and $\chi\left(s_{1}\right)=\chi\left(s_{2}\right)$. Thus $\chi\left(s_{1}\right) c^{n}=\chi\left(s_{2}\right) c^{m}$. We have shown that $\chi^{+}$is well-defined. But $\chi^{+}$is a homomorphism; hence $\chi^{+}$is a character of $\{S, x\}$ which extends $\chi$.

Case 2. Suppose that $\chi\left(x^{p}\right)=0$. Let $\chi^{+}$denote the function with domain $\{S, x\}$ defined by $s \rightarrow \chi(s)$ if $s \in S$ and by $s x^{n} \rightarrow 0$ if $s \in S$ and $n$ is an integer such that $0<n<p$. That $\chi^{+}$is well-defined can be shown by techniques similar to those used in case 1. $\chi^{+}$is a character of $\{S, x\}$ and is an extension of $\chi$.

Lemma 3. Let $T$ be a commutative semigroup. Assume that each character $\chi$ of each subsemigroup $S$ of $T$ has the property that $\chi(x s)=0$ whenever $x \in T / S$, $s \in S, x s \in S$, and $\chi(s)=0$. Then each character of each subsemigroup of $T$ can be extended to a character of $T$.

Proof. Suppose $\chi$ is a character of some subsemigroup $S$ of $T$. We show that $\chi$ can be extended to $T$. By Zorn's lemma there is a subsemigroup $S^{\prime}$ of $T$ which contains $S$ and which has the property that $\chi$ can be extended to $S^{\prime}$ but not beyond $S^{\prime}$. Assume that $\chi$ is defined on $S^{\prime}$. We show that $S^{\prime}=T$. Suppose that $x$ is a member of $T$ which is not in $S^{\prime}$. Define a function $\chi^{+}$on $\left\{S^{\prime}, x\right\}$ by $s \rightarrow \chi(s)$ if $s \in S^{\prime}$ and by $s x^{n} \rightarrow 0$ if $s \in S^{\prime}$ and $n$ is a positive integer. To show that $\chi^{+}$is well-defined it suffices to show that if $s x^{n}=s^{\prime}$ for $s, s^{\prime} \in S^{\prime}$ and $n$ a positive integer, then $\chi\left(s^{\prime}\right)=0$. Let $s, s^{\prime} \in S^{\prime}$ such that $s x^{n}=s^{\prime}$ for some $n$. Since $\chi$ cannot be extended beyond $S^{\prime}$, Lemma 2 implies that $x^{n} \notin S^{\prime}$. By Lemma 1 , it follows that $\chi(s)=0$. Thus $\chi\left(s^{\prime}\right)=0$ by hypothesis and $\chi^{+}$ is well-defined. It is easy to verify that $\chi^{+}$is a character which extends $\chi$. Thus the lemma follows.

Theorem 1. If $T$ is a commutative semigroup, then each character of each subsemigroup of $T$ can be extended to a character of $T$ if and only if each subsemigroup of each Archimedean component of $T$ is Archimedean.

Proof. Suppose first that each subsemigroup of each Archimedean component of $T$ is Archimedean and that there is a character of some subsemigroup of $T$ which cannot be extended to $T$. By Lemma 3 there exist a subsemigroup $S$ of $T$ and a character $\chi$ of $S$ with the property that there exist $x \in T / S$ and 
$s_{0} \in S$ such that $x s_{0} \in S, \chi\left(s_{0}\right)=0$, and $\chi\left(x s_{0}\right) \neq 0$. By Zorn's Lemma it is no loss of generality to assume that $\chi$ cannot be extended beyond $S$. Since $x s_{0}$ and $x^{2} s_{0}$ lie in the same Archimedean component $A$ of $T$, there exists $t \in A$ such that $x^{2} s_{0} t=\left(x s_{0}\right)^{n}$ for some positive integer $n$. That $x t \in S$ follows from Lemma 1 and the equation $\left(x s_{0}\right)(x t)=\left(x s_{0}\right)^{n}$. Similarly, the equation $\left(x s_{0}\right) t=s_{0}(x t)$ implies that $t \in S$. The semigroup $A \cap S$ is Archimedean, and both $x s_{0}$ and $t$ belong to $A \cap S$. Since $\chi\left(x s_{0}\right) \neq 0$, it follows that $\chi(t) \neq 0$. Lemma 1 now implies that $\chi$ can be extended to $\{S, x\}$ contrary to the choice of $S$.

Conversely, suppose that each character of each subsemigroup of $T$ can be extended to $T$ and that there exists an Archimedean component $A$ of $T$ and a subsemigroup $S$ of $A$ which is not Archimedean. It follows that there exist $s, s^{\prime} \in S$ such that $s S \cap\left\{s^{\prime}\right\}$ is void. Define a function $\chi$ from $s S \cup\left\{s^{\prime}\right\}$ into the multiplicative semigroup of complex numbers by $y \rightarrow 0$ if $y \in s S$ and by $y \rightarrow 1$ if $y \in\left\{s^{\prime}\right\}$. Then $\chi$ is a character of $s S \cup\left\{s^{\prime}\right\}$ and so has an extension $\chi^{+}$defined on $T$. Since $A$ is Archimedean, either $\chi^{+} \mid A$ is identically zero or $\chi^{+}(A)$ does not contain zero. The latter statement contradicts the way $\chi$ is defined on $s S \cup\left\{s^{\prime}\right\}$. The theorem follows.

Corollary 1. If $T$ is a commutative torsion semigroup, then each character of each subsemigroup of $T$ can be extended to a character of $T$.

Proof. Suppose $T$ is a torsion semigroup and that $S$ is a subsemigroup of some Archimedean component $A$ of $T$. If $x$ and $y$ are in $S$, then there exist positive integers $m$ and $n$ such that $x^{m}$ and $y^{n}$ are idempotent. Since $x^{n}$ and $y^{m}$ are in the same Archimedean component, $x^{n}=y^{m}$ (see (1), p. 135). It follows that each of $x$ and $y$ divides a power of the other in $S$. Thus $S$ is Archimedean and the corollary follows.

Note that if $x$ generates a free Abelian group, then the subsemigroup $\left\{x^{i} \mid i \geqq 0\right\}$ is not Archimedean. This remark along with the previous corollary yields:

Corollary 2. If $T$ is a commutative inverse semigroup, then each character of each subsemigroup of $T$ can be extended to a character of $T$ if and only if $T$ is torsion.

Note that a character $\chi$ of a commutative semigroup $S$ is a unit in the semigroup of all characters of $S$ if and only if $\chi$ vanishes at no point of $S$. Such characters will henceforth be called unit-characters. Ross has proved in (3) that a unit-character $\chi$ of a subsemigroup $S$ of a commutative semigroup $T$ can be extended to a unit-character of $T$ if and only if the equation $a x=a y$ implies that $\chi(x)=\chi(y)$ for $(a, x, y) \in T \times S \times S$. From this result, we obtain in the corollary to Theorem 2 a characterisation of those commutative inverse semigroups $T$ such that each unit-character of each subsemigroup of $T$ can be extended to a unit-character of $T$.

Theorem 2. If $T$ is a commutative, separative semigroup, then each unitcharacter of each subsemigroup of $T$ can be extended to a unit-character of $T$ if 
and only if $a x=$ ay implies $x=y$ for $a \in T$ and $x$ and $y$ in the same Archimedean component of $T$.

Proof. Assume $a x=a y$ implies $x=y$ for $a \in T$ and $x$ and $y$ in the same Archimedean component of $T$. Suppose there is a unit-character $\chi$ of some subsemigroup $S$ of $T$ such that $\chi$ cannot be extended to $T$. By Ross' theorem there exists $(a, z, w) \in T \times S \times S$ such that $a z=a w$ and $\chi(z) \neq \chi(w)$. Then $a z^{2} w=a w^{2} z$ and since $z^{2} w$ and $w^{2} z$ are in the same Archimedean component of $T$, it follows that $z^{2} w=w^{2} z$. Thus $\chi\left(z^{2} w\right)=\chi\left(w^{2} z\right)$ and $\chi(z)=\chi(w)$, which is a contradiction.

Conversely, suppose that each unit-character of each subsemigroup of $T$ can be extended to $T$ but that there exist distinct $x$ and $y$ in the same Archimedean component of $T$ such that $a x=a y$ for some $a \in T$. Since $T$ is separative, the Archimedean component of $T$ which contains $x$ and $y$ is cancellative (see (1), p. 133). Each commutative cancellative semigroup is a subsemigroup of some group. Moreover, the set of all group-characters of a group separate points of the group. (See (1), pp. 135 and 197.) Since each group-character of a group is a unit-character of the group, it follows that there is a unit-character $\chi$ of $\{x, y\}$ such that $\chi(x) \neq \chi(y)$. Since $\chi$ may be extended to $T$, we may assume that $\chi$ is defined on all of $T$. Since $\chi(a x)=\chi(a y)$, we have that $\chi(x)=\chi(y)$, and the theorem follows.

A subsemigroup $S$ of a commutative semigroup $T$ is said to be unitary in $T$ if the product $x s$ is in $S$ for $x \in T$ and $s \in S$ only if $x$ is also in $S$. If $T$ is a commutative inverse semigroup, then the subsemigroup of idempotents of $T$ is unitary in $T$ if and only if $a x=a y$ implies $x=y$, whenever $a \in T$ and $x$ and $y$ are in the same Archimedean component of $T$. Thus we have the following corollary of Theorem 2 .

Corollary. If $T$ is a commutative inverse semigroup, then each unit-character of each subsemigroup of $T$ can be extended to a unit-character of $T$, if and only if the subsemigroup $E_{T}$ of idempotents of $T$ is unitary in $T$.

\section{REFERENCES}

(1) A. H. Clifford and G. B. Preston, Algebraic theory of semigroups, Amer. Math. Soc. Math. Sur. 7 (1961).

(2) PAUL HILL, Characters of commutative semigroups, Journal of Algebra.

(3) K. A. Ross, Extending characters on semigroups, Proc. Amer. Math. Soc. 12 (1961).

(4) R. J. WARne and L. K. Williams, Characters on inverse semigroups, Czech. Math. Jour. 11 (1961).

\section{UNIVERSITY OF HOUSTON}

